\title{
Risk factors for poor outcome of surgery for cervical spondylotic myelopathy
}

\author{
JT Zhang, LF Wang, S Wang, J Li and Y Shen
}

Study design: Prospective study.

Objectives: The purpose of this study was to characterise risk factors for poor surgical outcome in patients with cervical spondylotic myelopathy (CSM).

Methods: The prospective study included 110 consecutive patients who underwent surgical treatment for CSM. Surgical outcomes were evaluated according to the Japanese Orthopaedic Association (JOA) score. JOA recovery rate $<50 \%$ was defined as poor surgical outcome. Relationship between outcome and various clinical and imaging predictors was examined. By multivariate logistic regression analysis, we identified risk factors associated with poor outcome. Receiver operating characteristic curves were plotted to acquire cutoff values for the continuous variables found to be independently associated with poor outcome.

Results: Forty-two patients (38.2\%) had a recovery rate of $<50 \%$. Logistic regression, with poor outcome as dependent variable, showed independent risks associated with increased age (odds ratio $(\mathrm{ORs})=1.08,95 \%$ confidence interval $(\mathrm{Cl})=1.01-1.15$, $P=0.021)$, symptom duration $(\mathrm{OR}=4.01,95 \% \mathrm{Cl}=1.95-8.23, P=0.000)$ and signal intensity ratio $(\mathrm{SIR}, \mathrm{OR}=4.24,95 \%$ $\mathrm{Cl}=1.61-11.20, P=0.003)$. The cutoffs with the best compromise between sensitivity and specificity were set at 63.1 years of age, 9 months of symptom duration and 1.455 for SIR. The presence of $\geqslant 2$ out of three factors (age $\geqslant 63.1$ years, symptom duration $\geqslant 9$ months and $\mathrm{SIR} \geqslant 1.455)$ gave an overall $\mathrm{OR}$ of $33.15(95 \% \mathrm{Cl}=4.11-267.37, P=0.001)$.

Conclusion: These findings suggest that advanced age, long-term CSM symptoms and high preoperative SIR are risk factors for poor outcome of surgery in patients with CSM.

Spinal Cord (2016) 54, 1127-1131; doi:10.1038/sc.2016.64; published online 3 May 2016

\section{INTRODUCTION}

Cervical spondylotic myelopathy (CSM) is a common cause of spinal cord dysfunction that generally requires surgical treatment owing to its progressive nature. ${ }^{1}$ The optimal treatment strategy of CSM is dependent on both progression and stage of disease. However, controversy remains regarding conservative versus surgical management, as favourable results have been reported for both. ${ }^{2}$ Patients with CSM have various symptoms such as sensory abnormality of the trunk or extremities, gait disturbance and urinary dysfunction. ${ }^{1,3}$ Although decompressive surgery is an available treatment option for this disease, the surgical outcome is not always satisfactory. Prognostic guidelines are still unclear, and it is very difficult for the surgeon to predict postoperative recovery.

Magnetic resonance imaging (MRI) is a valuable tool before surgical decompression because it allows the visualisation not only of the magnitude of spinal cord compression but also of intramedullary signal intensity. The presence of intramedullary increased signal intensity (ISI) on T2-weighted imaging (WI) in patients with CSM reflects chronic spinal cord compression. ${ }^{4-6}$ However, controversy exists in the reported results, mainly because of the lack of a proper quantitative assessment method of signal intensity changes in spinal cord. ${ }^{4,5,7-16}$ Wang et al. ${ }^{17}$ first used signal intensity ratio (SIR) as a quantifiable measure of signal intensity in cervical compressive myelopathy. Therefore, we hypothesised that there was a relationship between neurological function recovery after surgery and quantitative signal intensity on MRI. In addition to examining this relationship, we also designed the current study to identify risk factors associated with poor outcome, particularly the predictive value of quantitative SIR, after surgical treatment for CSM and provide insight into how surgeons undertake decision-making.

\section{MATERIALS AND METHODS}

\section{Ethics statement}

The study was approved by Ethics Committee of the Third Hospital of Hebei Medical University in China. All participants gave their informed consent to assessing and using their data. The methods were carried out in accordance with the approved guidelines.

\section{Patient population}

From January 2010 to December 2012, 266 patients with clinically diagnosed and imaging-confirmed CSM underwent surgical treatment at the Department of Spinal Surgery, the Third Hospital of Hebei Medical University in China. Operative indications for CSM patients were as follows: (1) up to three levels of anterior cord compression: anterior surgery; (2) more than three levels of anterior cord compression: posterior surgery; and (3) anterior and posterior cord compression: posterior with or without anterior surgery. Exclusion criteria were previous cervical spine surgery, vitamin B deficiency, rheumatoid arthritis, 
cervical ossification of the posterior longitudinal ligament, and concomitant lumbar spinal stenosis or other neurological disorders before their CSM surgery or during follow-up. A total of 110 patients with CSM who were followed for 12 months or more after surgery were prospectively enroled in this study. There were 63 men and 47 women, ranging in age from 38 to 81 years, with a mean age of 64.7 years. CSM was defined as a constellation of symptoms and signs supported by appropriate imaging studies, including routine radiographs, computed tomography and MRI.

All enroled patients underwent surgical decompression combined with instrumented fusion. Patients treated anteriorly underwent cervical discectomy and fusion, or cervical corpectomy and fusion. Posterior procedures included laminoplasty, or laminectomy and fusion. The surgical approach (anterior, posterior or a combination) and the number of operated segments were determined by one surgeon (YS). Data at the 12-month follow-up visit were used to assess important predictors of outcome, as 12 months represent a typical time period of optimum recovery after operation for CSM.

\section{Neurological assessment}

The preoperative and postoperative neurological function at 12 months of follow-up was assessed using the Japanese Orthopaedic Association (JOA) scoring system (Table 1). Postoperative improvement of symptoms was estimated on the basis of the recovery rate $=$ (postoperative JOA score preoperative JOA score $) /(17$-preoperative JOA score $) \times 100 \%$. A score of $75-100 \%$ was designated as excellent, $50-74 \%$ as good, $25-49 \%$ as fair and $0-24 \%$ as poor. Therefore, in this study, we defined a poor surgical outcome as a recovery rate $<50 \%$.

\section{Radiographic assessment}

Spinal alignment was measured on cervical spine radiographs. The C2-7 angle was measured between the posterior border of the $\mathrm{C} 2$ vertebral body and posterior border of the $\mathrm{C} 7$ vertebral body on lateral radiographs with patients in a neutral position. The Cobb method was used to measure the C2-7 range of motion through the change in the maximal flexion and extension by lateral radiographs.

All patients underwent preoperative high-resolution MRI with a 1.5-T system (Magnetom Symphony, Siemens Medical Solutions, Malvern, PA, USA). The MRIs of the spinal cord were obtained using a spin echo sequence system for T1-WI and a fast spin echo sequence system for T2-WI. The ISI values of the spinal cord on sagittal T2-WIs were obtained, and the regions of interest were taken by $0.05 \mathrm{~cm}^{2}$. The normal spinal cord signal intensity values on sagittal T2-WIs were obtained at the C7-T1 disc level, and the regions of interests were

Table 1 Comparison of patient characteristics between good and poor recovery groups

\begin{tabular}{lccr}
\hline Variable & Good $(\mathrm{n}=68)$ & Poor $(\mathrm{n}=42)$ & P-value \\
\hline Age at operation (years) & $62.4 \pm 9.1$ & $68.5 \pm 8.2$ & 0.001 \\
Female sex ( $n, \%)$ & $29(42.6 \%)$ & $18(43.0 \%)$ & 0.983 \\
BMI (kg m ${ }^{-2}$ ) & $25.7 \pm 3.1$ & $25.1 \pm 4.3$ & 0.385 \\
Diabetes mellitus ( $n$, \%) & $19(28.0 \%)$ & $8(19.0 \%)$ & 0.292 \\
Duration of symptoms (months) & $10.1 \pm 7.5$ & $20.7 \pm 15.6$ & $<0.001$ \\
Preoperative JOA score & $10.2 \pm 2.6$ & $9.6 \pm 2.2$ & 0.195 \\
JOA score at 12-month follow-up & $14.7 \pm 1.0$ & $11.8 \pm 2.3$ & $<0.001$ \\
Recovery rate (\%) & $66.9 \pm 10.4$ & $32.3 \pm 11.7$ & $<0.001$ \\
SIR & $1.29 \pm 0.17$ & $1.65 \pm 0.37$ & $<0.001$ \\
C2-7 angle (deg.) & $16.3 \pm 5.6$ & $15.6 \pm 6.3$ & 0.597 \\
C2-7 ROM (deg.) & $16.9 \pm 9.9$ & $17.3 \pm 6.1$ & 0.812 \\
Levels involved & $2.1 \pm 1.0$ & $2.0 \pm 1.1$ & 0.588 \\
Surgical approach & & & \\
$\quad$ Anterior & 41 & 31 & 0.334 \\
Posterior & 21 & 8 & \\
Combined anterior/posterior & 6 & 3 & \\
\hline
\end{tabular}

Abbreviations: BMI, body mass index; JOA, Japanese Orthopaedic Association; SIR, signal intensity ratio; ROM, range of motion. taken by $0.3 \mathrm{~cm}^{2}$. If no intramedullary ISI was noted on T2-WIs, the regions of interests were taken by $0.05 \mathrm{~cm}^{2}$ of the severely compressed cord. The SIR was defined as the signal intensity at the level of ISI or severely compressed cord (in cases with no ISI) divided by the signal intensity at the C7-T1 disc level. The signal intensity value was measured on the MRI workstation, and the SIR was calculated. The selection of the regions of interests was based on the balance of a number of factors. For instance, an extremely large area would not hold all patients in the group, whereas an extremely small area would jeopardise the accuracy of the signal intensity value.

\section{Statistical analysis}

Descriptive analysis of the patient population was conducted using means and standard deviations for continuous variables and frequencies and percentages for categorical variables. The duration of symptoms was estimated as the period from the onset of the primary neurological symptom to the time of surgery. Univariate analyses were performed to identify correlations between surgical outcome at 12 months of follow-up and prognostic factors. Comparison of continuous variables among the different groups was made using Student's $t$-test or the Mann-Whitney $U$-test, as appropriate. The categorical variables were compared by the $\chi^{2}$ test. Multivariate logistic regression analysis was also performed to control for potential confounding variables with the dependent variable of 'poor outcome'. Adjusted odds ratios (ORs) with 95\% confidence intervals (CIs) were presented with their respective $P$-values. Factors with a $P$-value $<0.05$ in univariate analysis were entered into the multivariate logistic model. The receiver operating characteristic curve analysis was constructed to evaluate the cutoff values for the continuous variables found to be independently associated with poor outcome. Finally, the logistic regression analysis was again used to test the association between poor outcome and the combination of variables with the best diagnostic accuracy for predicting poor outcome after adjusting for possible confounding factors. A value of $P<0.05$ was considered to represent a statistically significant difference. All analyses were performed using SPSS software (version 21.0; SPSS Inc., Chicago, IL, USA).

\section{RESULTS}

The range of SIR was 1.08-2.86 for all patients. Figure 1 illustrated the measurement of SIR on the MRI workstation. For all patients, the mean JOA score was 9.9 points preoperatively, and 13.6 points at 12 months postoperatively, yielding a mean recovery rate of $53.6 \%$. Thus, a statistically significant improvement in the JOA score was obtained at the 12-month follow-up $(P<0.001)$. Sixty-eight patients had good surgical outcomes, with recovery rates $\geqslant 50 \%$, whereas 42 patients had poor surgical outcomes with recovery rates $<50 \%$. Compared with the good-outcome group, the poor-outcome group had a significantly higher mean patient age $(P=0.001)$ and preoperative SIR on T2-WI $(P<0.001)$ and a significantly longer symptom duration $(P<0.001$, Table 1$)$. In multivariate logistic regression analysis, age $(\mathrm{OR}=1.08,95 \% \mathrm{CI}=1.01-1.15, P=0.020)$, symptom duration $(\mathrm{OR}=4.01,95 \% \mathrm{CI}=1.95-8.23, \quad P=0.000)$ and SIR $(\mathrm{OR}=4.24,95 \% \mathrm{CI}=1.61-11.20, \quad P=0.003)$ were independently associated with poor outcome (Table 2).

The receiver operating characteristic curve analysis showed that age, symptom duration and preoperative SIR, taken singly, had a good accuracy for predicting poor outcome (area under the curve: 0.702, 0.820 and 0.829 , respectively; $P=0.000, P=0.000, P=0.001$, respectively). The cutoffs with the best compromise between sensitivity and specificity were set at 63.1 years of age, 9 months of symptom duration and 1.455 for preoperative SIR (Figure 2 and Table 3). The presence of $\geqslant 2$ out of three factors (age $\geqslant 63.1$ years, symptom duration $\geqslant 9$ months and serum creatinine $\geqslant 1.455)$ was significantly associated with poor surgical outcome $(\mathrm{OR}=33.15,95 \% \mathrm{CI}=4.11-267.37$, $P=0.001$; Table 4). 


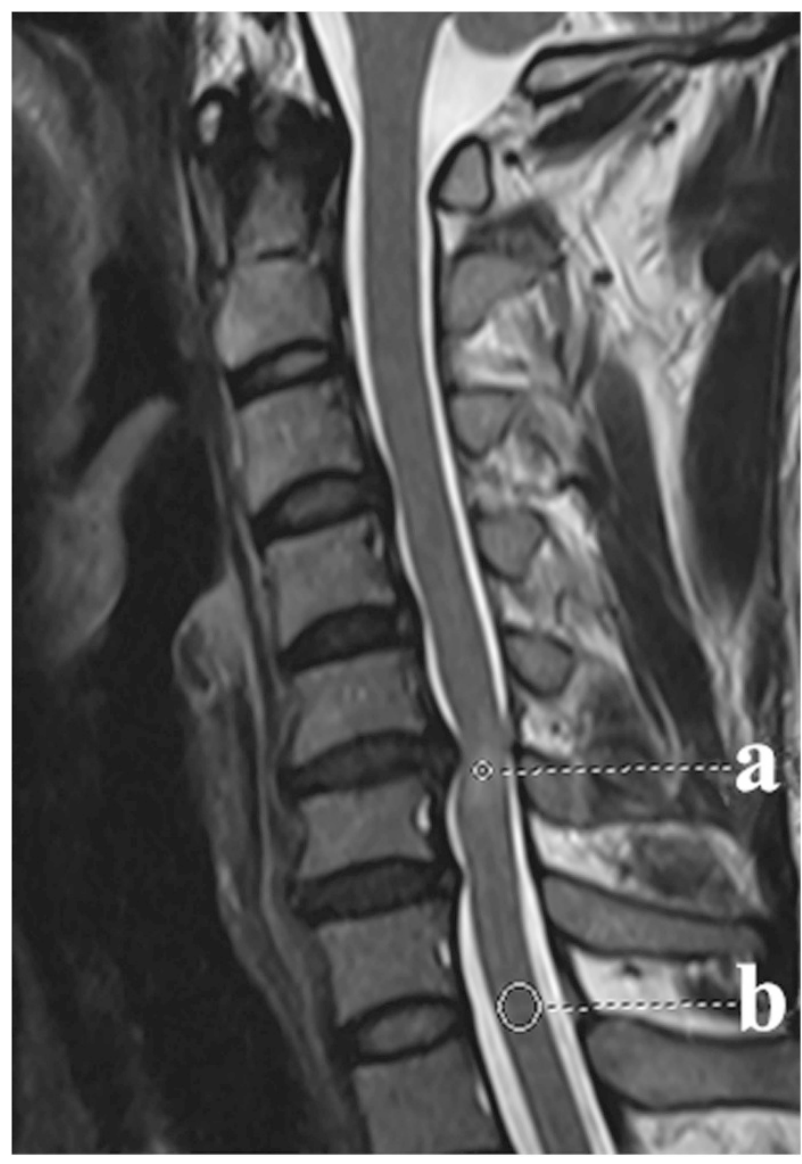

Figure 1 Measurement of SIR on MRI. SIR was defined as the signal intensity of ( $\mathbf{a}$ and $\mathbf{b}$ ).

Table 2 Risk factors for poor outcome after operation: multiple logistic regression analysis

\begin{tabular}{lcc}
\hline Variable $^{\mathrm{a}}$ & OR $(95 \% \mathrm{Cl})$ & P-value \\
\hline Age at operation (1-year increase) & $1.08(1.01-1.15)$ & 0.020 \\
Duration of symptoms (months) & $4.01(1.95-8.23)$ & 0.000 \\
SIR & $4.24(1.61-11.20)$ & 0.003
\end{tabular}

Abbreviations: $\mathrm{Cl}$, confidence interval; OR, odds ratio; SIR, signal intensity ratio.

aDuration of symptoms: $1 . \leqslant 3$ months; 2 . $>3$ but $\leqslant 6$ months; $3 .>6$ but $\leqslant 12$ months; 4 . $\geqslant 12$ but $\leqslant 24$ months; 5 . $>24$ months. SIR: $1 . \geqslant 1.00$ but $\leqslant 1.50 ; 2 .>1.50$ but $\leqslant 2.00 ; 3$. $>2.00$ but $\leqslant 2.50 ; 4$. $>2.50$.

\section{DISCUSSION}

Recognition of the best timing for surgery to ensure neurological improvement is an important clinical issue. Previous studies have shown that numerous factors affect postoperative outcomes of patients with CSM, including age, ${ }^{18}$ duration of CSM symptoms, ${ }^{7,10,18-21}$ signal changes on preoperative $\mathrm{MRI}^{5,6,11,13,22}$ and preoperative JOA score. ${ }^{23-25}$ However, the list of predictive factors differs according to researchers, and the prognostic significance of these factors remains controversial. In our study, we used a logistic regression model to determine the risk factors related to having a poor postoperative outcome. We demonstrated that patients with poor surgical outcome tended to have older age, longer duration of CSM symptoms and higher preoperative SIR than those patients with good outcome.

Previous studies have suggested that the spinal cord is vulnerable to the degeneration of motor neurons and myelinated fibres in elderly

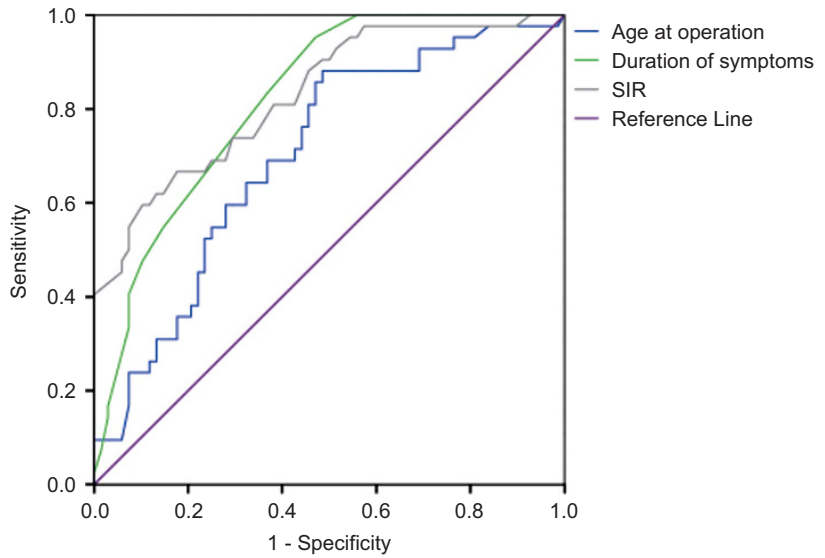

Figure 2 In receiver operating characteristic curves, the optimal cutoff values of age, duration of symptoms and SIR are shown for prediction of a poor surgical outcome.

Table 3 Sensitivity, specificity, AUC and cutoff of risk factors for predicting poor outcome

\begin{tabular}{lccccc}
\hline Variable & $S N$ & $S P$ & AUC & Cutoff & $P$-value \\
\hline Age at operation (years) & 0.857 & 0.529 & 0.702 & 63.1 & 0.000 \\
Duration of symptoms (months) & 0.925 & 0.529 & 0.820 & 9 & 0.000 \\
SIR & 0.667 & 0.824 & 0.829 & 1.455 & 0.001
\end{tabular}

Abbreviations: AUC, area under the curve; SN, sensitivity; SP, specificity; SIR, signal intensity ratio.

Table 4 Differences in the incidence of poor outcome after operation in patients with 0,1 or $\geqslant 2$ factors

\begin{tabular}{lcc}
\hline Factor & OR $(95 \% \mathrm{Cl})$ & P-value \\
\hline 0 factor & 1 & \\
1 factor & $1.10(0.09-12.99)$ & 0.942 \\
$\geqslant 2$ factors & $33.15(4.11-267.37)$ & 0.001 \\
\hline
\end{tabular}

Abbreviations: $\mathrm{Cl}$, confidence interval; $\mathrm{OR}$, odds ratio.

patients. $^{26,27}$ The results of the univariate analysis showed that patients in the poor-outcome group were significantly older than those in the good-outcome group $(P=0.001)$. Multivariate logistic analysis demonstrated that age was a predictor, and the odds of a poor outcome were 1.08 times greater for every 1-year increase in the patient age. This may be explained that the elderly experience age-related changes in the spinal cord including a decrease in the number of $\gamma$-motoneurons, number of anterior horn cells and number of myelinated fibres in the corticospinal tracts and posterior funiculus. In addition, general degeneration associated with the normal ageing process and increased risk of underlying diseases also have negative influence on surgical outcome. Although most surgeons will not discriminate on the basis of age, they should be aware that elderly patients may experience poor neurological recovery.

The duration of symptoms affects the severity and progression of the disease due to chronic compression by the lesions. Patients with poor surgical outcome for CSM were observed to have a longer duration of symptoms in the present study. The rationale is that 
chronic and long-standing compression of the spinal cord may lead to irreversible damage due to demyelination and necrosis of the grey matter. The longer the spinal cord is compressed by the lesions, the greater possibility of irreversible injury might exist. Therefore, to achieve the best results, surgical intervention should be undertaken as early as possible.

The utility of spinal cord MRI signal intensity has been widely studied, and various authors have speculated on its histopathologic significance and impact on surgical outcome. It has been reported that oedema, myelomalacia and gliosis involve ISI on T2-WI, suggesting irreversible changes of the spinal cord, and this signal intensity change was significantly associated with poor postoperative outcome of CSM. ${ }^{4,5,10-12}$ Intramedullary signal change on MRI is generally considered to reflect nerve tissue degeneration. These findings may range in severity from reversible cord oedema and ischaemia to irreversible cavitation and necrosis. Despite such evidence, many studies found no correlation between surgical outcome and intramedullary ISI on T2-WI. ${ }^{7,13-16,28,29}$ In the present study, SIR was the quantitative method used to assess the changes of signal intensity, as described in the previous study. ${ }^{17}$ The ISI on T2-WI is irregular in each scan, even when using the same MRI system, because the different sequence parameters are selected individually for each patient. Therefore, ISI on T2-WI is a wide-ranging variant that usually encompasses many levels of actual severity. Application of quantitative analysis can avoid possible judgment errors by each investigator. In the present study, high preoperative SIR can predict poor outcome after surgical treatment for patients with CSM. Specifically, the odds of a poor postoperative outcome were 4.24 times greater for every halfpoint increase in the preoperative SIR. We calculated that the optimal cutoff value of preoperative SIR as a predictor of poor postoperative outcome was 1.455 .

In the present study, sex, body mass index, diabetes mellitus and preoperative JOA score did not influence the outcome of the surgical intervention. The C2-7 angle, C2-7 ROM, the number of involved segments and surgical approach did not correlate with poor postoperative outcome. It is also uncertain whether these factors are predictive of surgical outcome, as our findings may be inconsistent with the results of previous studies. It remains to be seen, however, whether these factors are truly unrelated to surgical outcome. It is possible that statistical significance was not reached in this study because of the different statistical tests used across the different studies.

In the present study, the cutoffs used for age, symptom duration and preoperative SIR were assessed by receiver operating characteristic curve analysis. Another finding of interest is that patients with at least two out of three above-mentioned factors (age, symptom duration and preoperative SIR) were associated with 33.15-fold higher risk for poor surgical outcome compared with patients without risk factors. Whether this may be a useful clinical tool for indentifying CSM patients at risk for poor postoperative outcome deserves further study.

There are several limitations that need to be considered in our study. First, this was a single-centre study and involved only a limited number of CSM patients. Second, our follow-up term was 12 months after surgery, indicating that the relationship between the predictive factors and long-term outcome of neurological function could not be clearly established, although we could broadly predict the future condition from the trends observed. Third, although the JOA score is generally utilised, patient-reported outcomes were not considered in the study. Four, each high-resolution MRI system has different characteristic and working parameters, which may explain the differences between our results and those of previous studies.
Therefore, these limitations suggest that our findings require further validation of these results in larger patient samples.

\section{CONCLUSIONS}

The quantification of signal intensity on MRI for patients with CSM was used in the present study to assess the impact of intramedullary signal change on the surgical outcome. Advanced age, long-term CSM symptoms and high preoperative SIR are risk factors for poor outcome of surgery in patients with CSM. As persistent cord compression and disease progress may lead to a treatment failure, an understanding about the importance of predictive factors can help surgeons consider the indications of surgical treatment and evaluate the timing of surgery.

\section{CONFLICT OF INTEREST}

The authors declare no conflict of interest.

\section{ACKNOWLEDGEMENTS}

We thank Dr Ling De Kong for his assistance in the statistical analysis. No funds were received in support of this work.

\section{DATA ARCHIVING}

There were no data to deposit.

1 Clark CR. Cervical spondylotic myelopathy: history and physical findings. Spine (Phila Pa 1976) 1988; 13: 847-849.

2 Kadanka Z, Bednarik J, Novotny O, Urbanek I, Dusek L. Cervical spondylotic myelopathy: conservative versus surgical treatment after 10 years. Eur Spine J 2011; 20: 1533-1538.

3 Emery SE. Cervical spondylotic myelopathy: diagnosis and treatment. J Am Acad Orthop Surg 2001; 9: 376-388.

4 Mehalic TF, Pezzuti RT, Applebaum BI. Magnetic resonance imaging and cervical spondylotic myelopathy. Neurosurgery 1990; 26: 217-226; discussion 226-227.

5 Takahashi M, Yamashita Y, Sakamoto Y, Kojima R. Chronic cervical cord compression: clinical significance of increased signal intensity on MR images. Radiology 1989; 173: 219-224.

6 Takahashi M, Sakamoto Y, Miyawaki M, Bussaka H. Increased MR signal intensity secondary to chronic cervical cord compression. Neuroradiology 1987: 29: 550-556.

7 Wada E, Yonenobu K, Suzuki S, Kanazawa A, Ochi T. Can intramedullary signal change on magnetic resonance imaging predict surgical outcome in cervical spondylotic myelopathy. Spine (Phila Pa 1976) 1999; 24: 455-461; discussion 462.

8 Avadhani A, Rajasekaran S, Shetty AP. Comparison of prognostic value of different MRI classifications of signal intensity change in cervical spondylotic myelopathy. Spine J 2010; 10: 475-485.

9 de Rota JJF, Meschian S, de Rota AF, Urbano V, Baron M. Cervical spondylotic myelopathy due to chronic compression: the role of signal intensity changes in magnetic resonance images. J Neurosurg Spine 2007; 6: 17-22.

10 Okada Y, Ikata T, Yamada H, Sakamoto R, Katoh S. Magnetic resonance imaging study on the results of surgery for cervical compression myelopathy. Spine (Phila Pa 1976) 1993; 18: 2024-2029.

11 Matsuda Y, Miyazaki K, Tada K, Yasuda A, Nakayama T, Murakami H et al. Increased MR signal intensity due to cervical myelopathy. Analysis of 29 surgical cases. J Neurosurg 1991; 74: 887-892.

12 Suri A, Chabbra RP, Mehta VS, Gaikwad S, Pandey RM. Effect of intramedullary signal changes on the surgical outcome of patients with cervical spondylotic myelopathy. Spine J 2003; 3: 33-45.

13 Morio Y, Teshima R, Nagashima H, Nawata K, Yamasaki D, Nanjo Y. Correlation between operative outcomes of cervical compression myelopathy and mri of the spinal cord Spine (Phila Pa 1976) 2001; 26: 1238-1245.

14 Yone K, Sakou T, Yanase M, Ijiri K. Preoperative and postoperative magnetic resonance image evaluations of the spinal cord in cervical myelopathy. Spine (Phila Pa 1976) 1992; 17: S388-S392.

15 Alafifi T, Kern R, Clinical Fehlings M. and MRI predictors of outcome after surgical intervention for cervical spondylotic myelopathy. J Neuroimag 2007; 17: 315-322.

16 Mastronardi L, Elsawaf A, Roperto R, Bozzao A, Caroli M, Ferrante M et al. Prognostic relevance of the postoperative evolution of intramedullary spinal cord changes in signal intensity on magnetic resonance imaging after anterior decompression for cervical spondylotic myelopathy. J Neurosurg Spine 2007; 7: 615-622.

17 Wang LF, Zhang YZ, Shen Y, Su YL, Xu JX, Ding WY et al. Using the T2-weighted magnetic resonance imaging signal intensity ratio and clinical manifestations to assess the prognosis of patients with cervical ossification of the posterior longitudinal ligament. J Neurosurg Spine 2010; 13: 319-323. 
18 Fujiwara K, Yonenobu K, Ebara S, Yamashita K, Ono K. The prognosis of surgery for cervical compression myelopathy. An analysis of the factors involved. J Bone Joint Surg Br 1989; 71: 393-398.

19 Koyanagi T, Hirabayashi K, Satomi K, Toyama Y, Fujimura Y. Predictability of operative results of cervical compression myelopathy based on preoperative computed tomographic myelography. Spine (Phila Pa 1976) 1993; 18: 1958-1963.

20 Matsuyama Y, Kawakami N, Mimatsu K. Spinal cord expansion after decompression in cervical myelopathy. Investigation by computed tomography myelography and ultrasonography. Spine (Phila Pa 1976) 1995; 20: 1657-1663.

21 Pumberger M, Froemel D, Aichmair A, Hughes AP, Sama AA, Cammisa FP et al. Clinical predictors of surgical outcome in cervical spondylotic myelopathy: an analysis of 248 patients. Bone Joint J 2013; 95: 966-971.

22 Vedantam A, Rajshekhar V. Does the type of T2-weighted hyperintensity influence surgical outcome in patients with cervical spondylotic myelopathy? A review. Eur Spine J 2013; 22: 96-106.

23 Tetreault LA, Karpova A, Fehlings MG. Predictors of outcome in patients with degenerative cervical spondylotic myelopathy undergoing surgical treatment: results of a systematic review. Eur Spine J 2015; 24 (Suppl 2): 236-251.
24 Shin JJ, Jin BH, Kim KS, Cho YE, Cho WH. Intramedullary high signal intensity and neurological status as prognostic factors in cervical spondylotic myelopathy. Acta Neurochir (Wien) 2010; 152: 1687-1694.

25 Karpova A, Arun R, Davis AM, Kulkarni AV, Massicotte EM, Mikulis DJ et al. Predictors of surgical outcome in cervical spondylotic myelopathy. Spine (Phila Pa 1976) 2013; 38: 392-400.

26 Terao S, Sobue G, Hashizume Y, Shimada N, Mitsuma T. Age-related changes of the myelinated fibers in the human corticospinal tract: a quantitative analysis. Acta Neuropathol 1994; 88: 137-142.

27 Terao S, Sobue G, Hashizume Y, Li M, Inagaki T, Mitsuma T. Age-related changes in human spinal ventral horn cells with special reference to the loss of small neurons in the intermediate zone: a quantitative analysis. Acta Neuropathol 1996; 92: 109-114.

28 Chen CJ, Lyu RK, Lee ST, Wong YC, Wang LJ. Intramedullary high signal intensity on T2-weighted MR images in cervical spondylotic myelopathy: prediction of prognosis with type of intensity. Radiology 2001; 221: 789-794.

29 Okais N, Moussa R, Hage P. Value of increased MRI signal intensity in cervical arthrosis in myelopathies. Neurochirurgie 1997; 43: 285-290; discussion 290-291. 